activity seems to have no MHC restriction.

Does this new information on the nature, specificity, and genetic control of the NK cell allow any tentative conclusions as to its function in vivo? Certainly data exist correlating tumour incidence and the ability to reject tumour grafts with the level of NK activity. Moreover, there seems to be a reciprocal relationship between $T$ cell and NK activity, as athymic nude mice and thymectomised animals have higher levels of NK than intact syngeneic mice. The data from Kiessling's group, reported in this issue of Nature (page 174), showing that a subset of thymo- cytes can be killed by NK cells, and correlating the presence or absence of this subset with the level of NK activity, also suggests that regulation of NK and $T$ cells is closely connected. Should this subset of thymocytes turn out to be particularly susceptible to retravirus-induced transformation, then the surveillance and immunoregulatory theories could be neatly fused.

Peter Beverley is an Imperial Cancer Research Fund Staff Scientist and Dick Knight a Cancer Research Campaign Research Fellow at the ICRF Human Tumour Immunology Group, University College Hospital Medical School, London.

\title{
A two-dimensional superfluid?
}

\section{from P. V. C. McClintock}

HAS two-dimensional superfluidity been observed? There has been a continuing controversy as to whether or not this interesting phenomenon, predicted theoretically for ideal two-dimensional systems, has been seen in real liquid helium-4 films. Dash has argued persuasively (Phys. Rev. Lett. 41, 1178; 1978) that it has not; and that the experiments which had apparently demonstrated the effect were misinterpreted. Some new measurements now reported by Webster, Webster and Chester of the University of California at Los Angeles (Phys. Rev. Lett. 42, 243 ; 1979) seem, however, to have tilted the balance of the evidence back in the affirmative direction once more, in favour of the original interpretation.

The superfluidity of the "creeping film' which forms on surfaces dipping into liquid helium has, of course, been well known--and intensively studiedever since it was first discovered by Rollin in 1936. It is this phenomenon which is responsible for the famous demonstration of superfiuidity in which an open-topped cup of liquid helium is able to empty itself while remaining upright, using the film as a kind of syphon. The present interest centres, however, on very thin helium films: so thin, in fact, that no parameter can. change within their thickness, and hence nothing can happen in the dimension at right angles to the surface on which the film is adsorbed. Such films probably constitute what are effectively two-dimensional (2-D) systems.

The theory of 2-D helium has made rapid progress in recent years under the

P. V. C. McClintock is a Lecturer in the Department of Physics, University of Lancaster. stimulus, particularly, of the beautiful experimental work carried out by Dash and his co-workers at the University of Washington, using exfoliated graphite as a substrate. A striking prediction made by Nelson and Kosterlitz (Phys. Rev. Lett. 39, 1201; 1977) was that, if a 2-D system undergoes a superfluid transition, it will do so in a precipitous jump: the superfluid fraction ought to jump discontinuously from zero to a finite value, $\rho_{\mathrm{B}}^{*}$. This is in sharp contrast to what happens in three dimensions. When ordinary bulk liquid helium undergoes the transition, the fraction of the liquid which behaves as superfluid increases continuously from zero. Nelson and Kosterlitz concluded, furthermore, that for a 2-D liquid helium-4 film the jump should be universal: that is, the ratio of $\rho_{\mathrm{s}}^{0}$ to the absolute temperature $T_{r}$ at onset should be quite independent of the nature of the substrate, of the coverage at which the transition takes place, or indeed, of virtually anything else.

Apparent experimental verification of this remarkable prediction was reported shortly afterwards: by Rudnick, on the basis of third sound measurements (Phys. Rev. Lett. 40, 1454; 1978); and by Bishop and Reppy, based on their measurements of the superfluid fraction on a vibrating substrate (Phys. Rev. Lett. 40, 1727; 1978). The latter type of measurement seems at first sight to give a particularly direct and unambiguous measure of the superfluid fraction. The period of the oscillator is dependent on its mass, and thus on the effective mass of the adsorbed helium film sticking to it; but, if some proportion of the film is superfluid then, instead of following the oscillations, this fraction will just slip, and therefore will not affect the measured period. Values of $\rho^{\circ} / T_{\mathrm{c}}$ deduced in this way were in excellent quantitative agreement with Nelson and Kosterlitz's prediction.

Dash argued, however, that the superfluid onset as measured by this technique does not represent genuine 2-D superfluidity. He suggested, first, that inhomogeneities in the Mylar substrate would cause the formation of 'puddles' of helium where the film was relatively thick, with interconnecting regions of thinner film. The observed superfluid onset therefore represented, not 2-D superfluidity in the film as a whole, but merely the occurrence of superfluidity in a sufficient proportion of the interconnecting regions. By this time the deep 'puddles' would, of course, correspond to 3-D superfluid. Second, he suggested that even if the substrate did provide an ideal atomically flat surface (which he doubted), the films themselves would be nonuniform. He envisaged that, as helium atoms were gradually added to the substrate, at a fixed temperature, they would first form a 2-D gas; but that, when a certain critical coverage was reached, interactions between the atoms would result in the formation of small patches of 2-D liquid surrounded by the 2-D vapour. As further atoms were added the connectivity of the liquid regions would eventually provide a continuous 2-D path across the whole experimental area and, if the temperature was low enough, superfluidity might then appear abruptly: the superfluid fraction at onset could clearly be non-zero, corresponding in fact to that of the liquid regions immediately before their being connected together.

In the experiment now reported from UCLA, the Websters and Chester have performed another vibrating substrate measurement, using an oscillating quartz crystal. The important difference from earlier work is that they have added an additional variable parameter to the system by mixing up to $30 \%$ of helium-3 with their helium-4: a proportion of helium-3 has a profound effect on the superfluid transition in helium-4, depressing it to lower temperatures and even eliminating it altogether if too much is added. Nonetheless, it turned out that the value of $\rho: / T_{\mathrm{c}}$ was completely unaffected by the helium -3 , remaining closely equal to the universal constant predicted by Nelson and Kosterlitz-although, of course, $\rho$ and $T_{c}$ were individually changed to a considerable degree. So, for some as yet unexplained reason, Dash's arguments do not seem to be applicable in practice.

These latest results constitute compelling evidence in support both of the theory and of the belief that the helium-4 film at onset really does behave as a two-dimensional superfluid. 